\title{
Tendência de mortalidade por lesões autoprovocadas intencionalmente no Brasil no período de 2004 a 2014
}

\author{
Mortality trend due to intentionally self-inflicted \\ injuries in Brazil from 2004 to 2014 \\ Lélia Lessa Teixeira Pinto', Saulo Sacramento Meira', Ícaro José Santos Ribeiro', Adriana Alves Nery', \\ Cezar Augusto Casotti
}

\section{RESUMO}

Objetivo: Analisar as tendências de mortalidade por lesões autoprovocadas intencionalmente segundo as faixas etárias e regiões do Brasil. Métodos: Trata-se de um estudo epidemiológico, de desenho ecológico do tipo série temporal, utilizando dados do Sistema de Informação sobre Mortalidade relacionada aos óbitos por lesões autoprovocadas intencionalmente ocorridos no período de 2004 a 2014. Resultados: A análise de tendência indicou crescimento dos coeficientes de mortalidade, sendo que a curva do Brasil acentuou a elevação $\left(R^{2}=0,678\right)$. A região Sudeste mostrou a maior inclinação de reta $\left(R^{2}=0,960\right)$, sendo superior mesmo a tendência observada no Brasil. Os coeficientes de mortalidade segundo faixas etárias evidenciaram tendência crescente para a faixa de 10 a 19 anos $\left(R^{2}=0,429\right)$. No grupo etário de 20 a 29 anos no período analisado, não houve tendência de crescimento. Entretanto, houve incremento do número de óbitos a partir do ano de 2010. Procedeu-se avaliação desse grupo no período de 2010-2014, sendo assim evidenciada tendência crescente para a referida faixa $\left(R^{2}=0,927\right)$. Conclusão: Os resultados apontam o crescimento dos coeficientes de mortalidade por lesões autoprovocadas intencionalmente no Brasil e em todas as suas regiões, sendo o grupo etário mais vulnerável o de 10 a 19 anos, seguido da faixa etária de 20

\section{Palavras-chave \\ Mortalidade, tentativa de suicídio, saúde coletiva.}

a 59 anos e de maiores de 60 anos. 


\section{Keywords}

Mortality, suicide attempted, collective health. coefficients by self-inflicted injuries in Brazil and in all its regions, with the age group being the most understood 10 to 19 years old, followed by the age group of 20 to 59 years and those over 60 years. Conclusion: The results point to the increase in mortality coefficients due to intentional self-inflicted injuries in Brazil and all regions, the most vulnerable age group being 10 to 19 years old, followed by the age group of 20 to 59 years and those over 60 .

\section{INTRODUÇÃO}

A violência pode ser classificada em três categorias: interpessoal, coletiva e autoinfligida, podendo estas resultar em ferimento, morte, dano psicológico, mal desenvolvimento ou danos sociais'. Esta última, denominada também como lesão autoprovocada intencionalmente (LAI), compreende comportamentos violentos incluindo ato suicida e autoagressão. O primeiro abrange pensamentos suicidas, tentativas de suicídio e suicídios concluídos, ou seja, em que há evidências implícita ou explícita da intenção de morte; o segundo envolve atos de automutilação, em que a intenção não é matar-se ${ }^{1,2}$. A Classificação Internacional de Doenças e Problemas Relacionados à Saúde (CID-10) estabelece como um dos tipos de causas externas de morbimortalidade as lesões autoprovocadas intencionalmente, sendo essas as lesões ou o envenenamento autoinfligido intencionalmente e as tentativas de suicídio³.

Entre as lesões autoprovocadas intencionalmente, o suicídio representa um grave problema de saúde pública, pois, segundo a Organização Mundial de Saúde (OMS), no ano de 2012, estima-se que ocorreram 804.000 óbitos por suicídio em todo o mundo ${ }^{4}$. No Brasil, no ano de 2011, o suicídio representou 6,8\% das mortes por todas causas externas, sendo mais frequente em homens, idosos do sexo masculino, com baixa escolaridade e a cor da pele ou raça parda 5 .

Entre as regiões brasileiras, a mortalidade por suicídio comporta-se de forma distinta. Estudo realizado com o objetivo de analisar a mortalidade por suicídio no Brasil no período de 2000 a 2012 identificou crescimento desse indicador, sendo o maior coeficiente identificado na região Sul do país $(9,8 / 100.000)$ e o maior crescimento percentual, na região Nordeste $(72,4 \%)^{6}$.

Diversos fatores de risco atuam diretamente aumentando a vulnerabilidade ao comportamento suicida, como os fatores psicológicos, biológicos, econômicos e socioculturais. Entre eles, as dificuldades como acesso e atendimento aos cuidados de saúde, facilidade aos meios de acesso de suicídios e mídia influenciadora, violências, relações sociais conflituosas, transtornos mentais, abuso de substâncias nocivas e problemas financeiros ${ }^{4}$.

Com isso, identificar fatores de risco individuais que estimulam o comportamento suicida é de suma relevância para explicar a opção do indivíduo, no entanto, não é suficiente para compreender as variações nas taxas de suicídio entre as regiões. O fato de conhecer as particularidades locais pode promover ações de proteção e prevenção, consequentemente impactar na redução da mortalidade por suicídio. Sendo considerados os óbitos por LAI preveníveis, estes desencadeiam sofrimento material e psicológico aos indivíduos que fazem parte das relações pessoais e sociais da vítima; prejuízo socioeconômico, pois, uma parcela das vítimas são indivíduos economicamente ativos; e incremento nos gastos do sistema de saúde, como atendimento ambulatorial, emergência, tratamento de sequelas dos sobreviventes ${ }^{6}$.

Como estudos de tendência temporal têm uma relevância epidemiológica, compreender o fenômeno e a distribuição deste pode auxiliar na determinação de hipóteses explicativas e na avaliação indireta da efetividade das políticas públicas ${ }^{7}$. Este estudo teve como objetivo analisar as tendências de mortalidade por lesões autoprovocadas intencionalmente, segundo as faixas etárias e regiões do Brasil.

\section{MÉTODOS}

Trata-se de um estudo epidemiológico, de desenho ecológico do tipo série temporal, utilizando dados secundários relacionados aos óbitos por lesões autoprovocadas intencionalmente referentes ao período de 11 anos (2004 e 2014).

Foram selecionados os óbitos registrados no Sistema de Informação sobre Mortalidade (SIM), compreendendo as causas referentes aos códigos de X60 a X84 da 10a Classificação Internacional de Doenças (CID-10). Optou-se pela estratificação dos dados de acordo com as faixas etárias de 10 a 19 anos, 20 a 59 anos e igual ou superior a 60 anos, e por regiões do Brasil. Para fins do cálculo dos coeficientes de mortalidade nos estratos, as informações populacionais foram obtidas no site do Instituto Brasileiro de Geografia e Estatística.

Para todas as medidas epidemiológicas estimadas, dados relativos à incidência de óbitos foram plotados e, por meio de gráficos de linhas, verificou-se o coeficiente de determinação $\left(R^{2}\right)$. Para a realização do processo de modelagem, as taxas padronizadas de mortalidade foram analisadas como variável dependente (y), e os anos do estudo, como variável independente (x). Para a análise da tendência, optou-se pela escolha do melhor modelo baseada no nível de significância (p) e na análise de resíduos. A significância estatística do modelo de tendência foi admitida ao se obter $p<0,05$. 
A análise dos dados deste estudo foi realizada com o auxílio do software Statistical Package for the Social Sciences (SPSS) versão 22.0.

\section{RESULTADOS}

Os coeficientes de mortalidade por LAl tiveram contínuo crescimento no país (4,6/100 mil em 2004 para 5,7/100 mil em 2014). Em números absolutos, registraram-se 8.017 óbitos em 2004 contra 10.653 em 2014. A Figura 1 ilustra a flutuação temporal do coeficiente de mortalidade para as LAI.

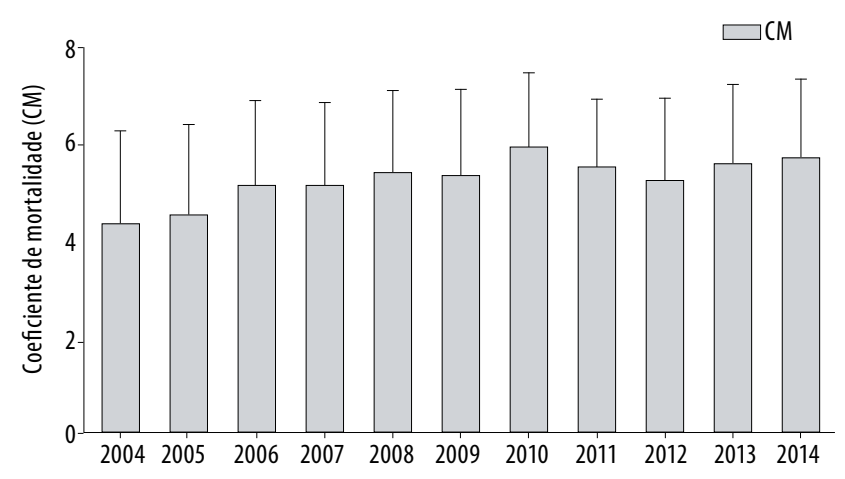

Figura 1. Média e desvio-padrão dos coeficientes de mortalidade por LAl de 2004 a 2014 no Brasil. Brasil, 2016.

A análise de tendência (Tabela 1) indicou crescimento dos coeficientes de mortalidade por LAl, sendo que a curva do Brasil acentuou a elevação $\left(R^{2}=0,678 ; p<0,005\right)$. Ao comparar a estratificação pelas regiões, o Sudeste mostrou a maior inclinação de reta $\left(R^{2}=0,960 ; p<0,001\right)$, sendo superior mesmo a tendência observada no Brasil.

A observância dos coeficientes de mortalidade por LAI de acordo com as faixas etárias (Tabela 1) evidenciou au- sência de tendência para as categorias de 20 a 59 anos e 60 anos a mais. $O$ único grupo etário que apresentou tendência crescente foi o de 10 a 19 anos $\left(R^{2}=0,429 ; p<0,05\right)$. Todavia, notado um incremento do número de óbitos a partir do ano de 2010 para a faixa etária de 20 a 59 anos, optou-se por proceder avaliação do período de 2010-2014, tendo sido evidenciada tendência crescente para a referida faixa $\left(R^{2}=\right.$ $0,927 ; p<0,005)$.

Nota-se que entre as faixas etárias analisadas, ilustradas na Figura 2, a maior oscilação foi evidenciada na categoria de 20 a 59 anos, de 6,65/100 mil em 2004 para 7,09/100 mil em 2014. Por outro lado, a menor oscilação foi identificada na faixa de 60 anos ou mais, variando de 7,46/100 mil em 2004 para 7,30/100 mil em 2014.

Aumento progressivo dos óbitos por LAl foi observado no Brasil e nas regiões do país. A maior e a menor oscilação foram observadas, respectivamente, na região Sudoeste de 3,51/100 mil em 2004 para 5,03/100 mil em 2014 e na região Sul de 7,41/100 mil em 2004 para 7,99/100 mil em 2014 (Figura 3).

\section{DISCUSSÃO}

Os resultados deste estudo indicam que o coeficiente de mortalidade por LAI no Brasil apresentou tendência crescente no período analisado, avançando de 4,56/100 mil em 2004, alcançando 5,73/100 mil em 2014. Conforme evidenciado, houve crescimento do coeficiente em todo o território nacional, levando-o a constituir-se como uma importante questão de saúde pública.

Ao compararmos com coeficientes de estudo anterior, notam-se estabilidade entre 1980 e 1994 (4,5/100 mil) e aumento no triênio 1995-1997 (5,4/100 mil), permanecendo estáveis até $2006^{8}$. Apesar de ainda possuir coeficientes relativamente baixos, por ser um país populoso, o Brasil está entre os dez países que registram os maiores números absolutos de suicídios, segundo dados da OMS ${ }^{4-9}$.

Tabela 1. Tendência nos coeficientes de mortalidade por LAI. Brasil e Regiões, 2004-2014

\begin{tabular}{|c|c|c|c|c|}
\hline & Modelo & $R^{2}$ & $P$ & Tendência \\
\hline Brasil & $y=-0,0148 x^{2}+0,2775 x+4,3092$ & 0,678 & $<0,005$ & Crescente \\
\hline \multicolumn{5}{|l|}{ Regiões } \\
\hline Norte & $y=-0,0065 x^{2}+0,2564 x+2,2544$ & 0,917 & $<0,001$ & Crescente \\
\hline Nordeste & $y=-0,0084 x^{2}+0,2207 x+2,8843$ & 0,806 & $<0,001$ & Crescente \\
\hline Sudeste & $y=0,0028 x^{2}+0,1077 x+3,4512$ & 0,960 & $<0,001$ & Crescente \\
\hline Sul & $y=0,006 x^{2}-0,0082 x+7,4587$ & 0,444 & $<0,05$ & Crescente \\
\hline Centro-0este & $y=0,0154 x^{2}-0,0569 x+5,1344$ & 0,686 & $<0,005$ & Crescente \\
\hline \multicolumn{5}{|l|}{ Faixa etária } \\
\hline 10 a 19 anos & $y=-0,0016 x^{2}+0,0852 x+1,6133$ & 0,429 & $<0,05$ & Crescente \\
\hline 20 a 59 anos & $y=0,0242 x^{2}-0,2561 x+6,7364$ & 0,092 & $>0,05$ & \\
\hline 60 ou mais & $y=0,0031 x^{2}+0,0192 x+7,0086$ & 0,057 & $>0,05$ & \\
\hline
\end{tabular}




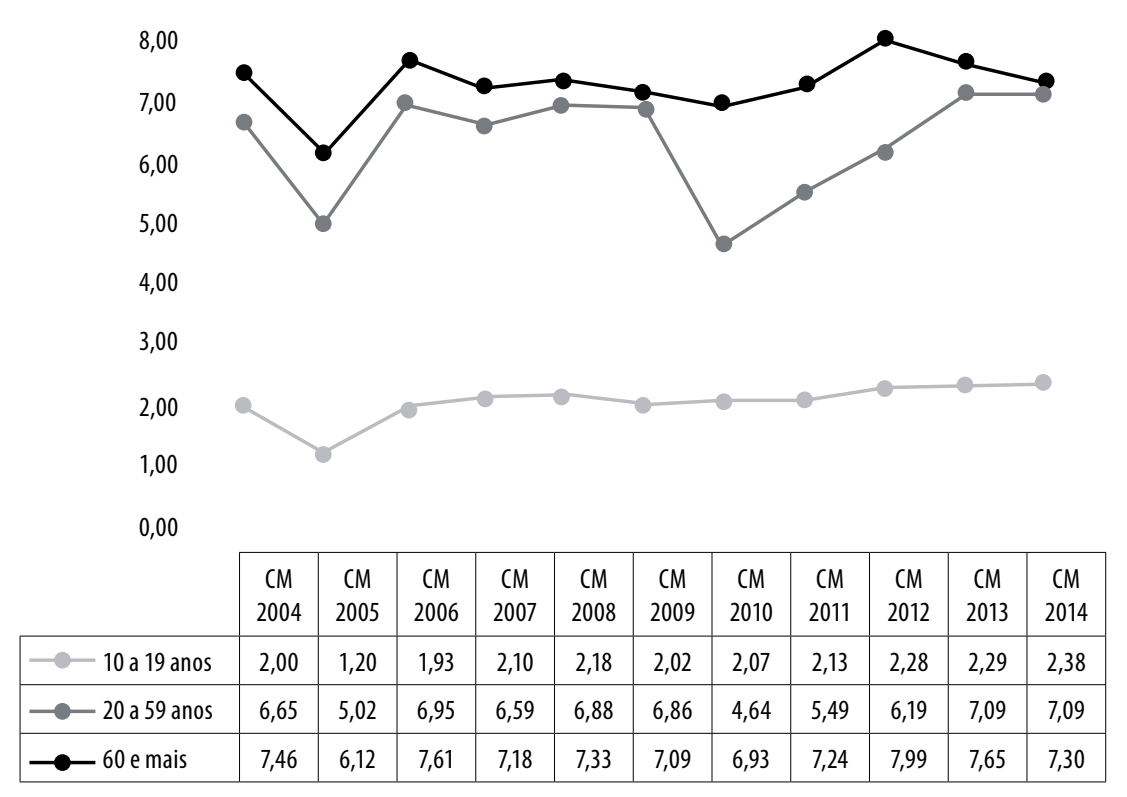

Figura 2. Coeficientes de mortalidade por LAI, segundo faixas etárias. Brasil, 2004-2014.

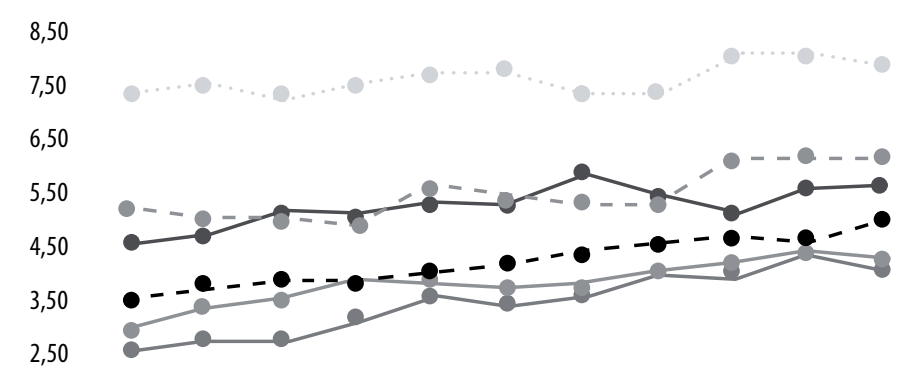

1,50

\begin{tabular}{|c|c|c|c|c|c|c|c|c|c|c|c|}
\hline & 2004 & 2005 & 2006 & 2007 & 2008 & 2009 & 2010 & 2011 & 2012 & 2013 & 2014 \\
\hline$\longrightarrow$ Brasil & 4,56 & 4,73 & 5,12 & 5,13 & 5,37 & 5,33 & 5,91 & 5,52 & 5,23 & 5,61 & 5,73 \\
\hline$\longrightarrow-$ CM Norte & 2,59 & 2,76 & 2,77 & 3,14 & 3,59 & 3,44 & 3,61 & 4,01 & 4,02 & 4,4 & 4,1 \\
\hline$\longrightarrow$ CM Nordeste & 2,94 & 3,37 & 3,49 & 3,86 & 3,92 & 3,74 & 3,78 & 4,09 & 4,16 & 4,44 & 4,26 \\
\hline - - - CM Sudeste & 3,51 & 3,77 & 3,88 & 3,79 & 4 & 4,19 & 4,39 & 4,58 & 4,7 & 4,65 & 5,03 \\
\hline ....... CM Sul & 7,41 & 7,57 & 7,34 & 7,53 & 7,74 & 7,85 & 7,42 & 7,43 & 8,12 & 8,15 & 7,99 \\
\hline - - CM Centro-0este & 5,15 & 5,07 & 5,02 & 4,91 & 5,63 & 5,46 & 5,34 & 5,3 & 6,12 & 6,28 & 6,24 \\
\hline
\end{tabular}

Figura 3. Coeficientes de mortalidade por LAl, segundo regiões do Brasil, 2004-2014.

É importante salientar que coeficientes nacionais de mortalidade por suicídio escondem importantes variações regionais, em especial no Brasil, que apresenta ao longo do seu processo de construção socioeconômico elementos pluriculturais profundos e complexos e que devem ser considerados para a compressão das lesões autoprovocadas. No âmbito das ciências sociais, o estudo de Durkheim ${ }^{10}$ considera que o suicídio está associado a fatos sociais que transcendem a esfera pessoal, estabelecendo a compreensão de que um evento que parecia depender unicamente de fatores individuais e psicológicos poderia expressar dissoluções na solidariedade social. A questão subjacente, portanto, é que relações podem ser estabelecidas entre as formas de vida social e os atos individuais voltados para abandoná-la.

As regiões compostas por imigrantes europeus merecem destaque porque apresentam maior número de suicídios, se comparados a outras regiões. Historicamente, a região Sul do Brasil vem apresentando os maiores coeficientes de suicídio do país. Esse estudo confirmou o elevado coeficiente de mortalidade por lesões autoprovocadas intencionalmente que se mantém com pouca variação no período estudado. Esse fato tem instigado pesquisadores oriundos de vários campos do conhecimento, destacando-se as ciências sociais e da saúde, que apontaram a etnia, a cultura tipicamente 
europeia, as crises sociais e inclusive aspectos climáticos da região como possíveis fatores ligados ao problema"1.

Esses valores ainda podem estar relacionados com a composição social, historicamente composta por agricultores, tendo sido uma das primeiras fronteiras agrícolas fechadas do país. Quando o suicídio acontece preponderantemente em um grupo etário, étnico, profissional ou isolado geograficamente, pode-se indagar se esse evento funcionasse como barômetro indicador de pressão na sociedade. A mortalidade elevada de suicídio em agricultores estaria refletindo as precárias condições de sobrevivência desse estrato populacional, endividamento, concentração da terra, êxodo e anomia; ou exposição profissional intensa aos agrotóxicos, que pode acarretar quadros depressivos desencadeados por mecanismos neurológicos ou endócrinos, podendo causar quadros de intoxicação aguda ou crônica' ${ }^{12,13}$.

Algumas substâncias podem afetar o sistema nervoso central, provocando transtornos psiquiátricos como ansiedade, irritabilidade, insônia ou sono conturbado (com excesso de sonhos e/ou pesadelos), quadros de depressão e, muitas vezes, levando a pessoa intoxicada ao ato extremo de eliminar a própria vida em decorrência da manipulação dos agrotóxicos. Estes são absorvidos pelo corpo humano pelas vias respiratória e dérmica e, em menor quantidade, também pela via oral14. O uso de venenos, em especial o organofosforado e o manganês, exposto em curto ou a longo tempo, deixa sequelas neurocomportamentais que podem evoluir para um quadro de depressão, sendo esse um forte fator de risco para o suicídio. A ação tóxica do primeiro agrotóxico age na inibição da colinesterase ${ }^{15}$, já o manganês age diretamente no sistema nervoso central, provocando também tremores e outros sintomas semelhantes aos do mal de Parkinson. Esse quadro, aliado a uma série de problemas econômicos e sociais, poderia levar ao suicídio ${ }^{14}$.

Doenças psiquiátricas, especialmente distúrbios de depressão, ansiedade e uso indevido de álcool, são fatores de risco bem conhecidos para lesões autoprovocadas. Todavia, as associações entre doenças físicas e lesões autoprovocadas são menos bem estabelecidas, particularmente em jovens. Estudos anteriores em adultos demonstraram associação entre comportamentos suicidas e doenças físicas crônicas, como asma, diabetes mellitus tipo I, epilepsia e câncer ${ }^{16}$.

Um estudo acerca da saúde mental de agricultores da Serra Gaúcha apontou para transtornos psiquiátricos em colonos das lavouras de uva e maçã, revelando que indivíduos que apresentaram alguma intoxicação aguda por agrotóxico tinham três vezes mais distúrbios psiquiátricos ${ }^{17}$.

A tendência de crescimento de lesões autoprovocadas nas regiões Norte e Centro-Oeste alerta para possíveis fatores causais, como, por exemplo, a maior concentração nessas regiões de grupos populacionais como o de indígenas, cujos coeficientes de mortalidade aproximam-se dos de países do Leste Europeu e da Escandinávia, na casa dos 15-30/100 mil, na medida em que alguns fatores, como o uso abusivo de bebidas alcoólicas e a elevada frequência de sofrimento mental, parecem se associar a esses altos índices ${ }^{18-20}$.

O processo de desvalorização da cultura indígena ao longo da história do Brasil, com a exaltação de estilos de vida cada vez mais tecnológico e capitalista, parece contribuir para a elevação dessas taxas, na proporção que as tribos indígenas passam a ser urbanizadas, porém sem chances de inserção ocupacional e sem garantia de sobrevivência.

O "não espaço" na sociedade aumenta o sentimento de não pertencimento ao local onde vivem, diminuindo a autoestima, a autovalorização e a admiração por seus pares. Sabe-se que os indígenas enfrentaram, ao longo da história, condições de vida e saúde mais precárias do que a população geral, além de sofrerem com a redução das terras e populações indígenas; isso tudo influencia a alta mortalidade por suicídio entre os indígenas no país ${ }^{20}$. Nessas situações, são comuns sintomas de fadiga, irritabilidade, baixas autoestima e autoconfiança, visões sombrias e pessimistas do futuro, ideias de culpa e desvalia e de autoagressão. Quando a depressão ocorre concomitante com o alcoolismo, resulta em um quadro ainda mais grave, aumentando o risco de suicídio. A depressão é uma das comorbidades que acompanham com maior frequência o diagnóstico de alcoolismo: 70\% dos suicidas têm depressão maior ${ }^{21}$.

O alcoolismo é apontado por estudiosos como um fator de influência significativo e muito presente na maior parte dessas comunidades. Em relatos da época do "descobrimento", a atração dos indígenas pelo álcool foi fortemente documentada, uma vez que se tornava um mecanismo de aliciamento e dominação dos povos nativos. O aumento dos casos de alcoolismo e depressão em grupos indígenas tem elevado a ocorrência da taxa de mortalidade dos índios brasileiros em três a quatro vezes em relação à média nacional (sociedade nacional envolvente), dependendo do Estado da federação. O alcoolismo tem sido, portanto, considerado uma das principais causas de mortalidade, seja pelo agravo de doenças como cirrose, diabetes, hipertensão arterial, doenças do coração, do aparelho digestivo, depressão e estresse ou como causa de morte por fatores externos como acidentes, brigas, quedas, atropelamentos, entre outros ${ }^{22}$.

O ritmo ascendente apresentado pela região Sudeste na série histórica corrobora com um estudo realizado na Hungria que oferece uma noção mais específica de fatores que podem estar envolvidos no risco associado as ideias, tentativas e concretizações suicidas. Com efeito, Almasi et al. ${ }^{23}$ sugerem que os maiores fatores de risco tendem a estar associados a fatores sociodemográficos, baixa escolaridade, estado civil, bem como a isolamento social24, características essas que se acentuam na região mais rica e populosa do Brasil, apontando para incoerências geradas pelas estruturação social.

Embora a região Sudeste ofereça mais postos de trabaIho, nelas o cotidiano é mais difícil e o custo de vida elevado. 
As condições precárias de trabalho, os baixos salários, a dificuldade no transporte, os longos trajetos e a falta de equipamentos urbanos trazem efeitos desastrosos para a saúde física e mental dos indivíduos. Segundo Dejours ${ }^{25}$, na cidade de São Paulo foi possível observar associação inversa do suicídio com desemprego, embora essa relação não seja significativa. Isso indica que a sobrecarga e o sofrimento emocional relacionado ao trabalho atingem tal magnitude que o risco do trabalhador ao suicídio é maior que o dos desempregados.

No que se refere à tendência crescente de lesões autoprovocadas intencionalmente na região Nordeste, Teixeira et al. ${ }^{26}$ afirmam que o Brasil vivencia uma potencial situação de risco em todo seu território, o que coloca o país em uma posição de vulnerabilidade diante dos interesses da indústria de agrotóxicos, seja em decorrência de ausência de um monitoramento da oferta e do consumo de produtos ou da conscientização acerca das complicações para a saúde advindas de seu uso.

Uma hipótese plausível para esse fenômeno poderia ser uma melhora na qualidade dos registros, especialmente em regiões menos desenvolvidas. Em geral, os dados de mortalidade nos países subdesenvolvidos tendem a ser deficientes, enquanto nos países desenvolvidos alcançam níveis confiáveis ${ }^{27}$. No Brasil, a análise da qualidade dos dados de 2000 a 2002 mostrou que apenas 5\% dos municípios do Nordeste apresentaram um nível satisfatório de registros de óbitos, nascimentos e causas mal definidas, enquanto $63 \%$ dos municípios do Sul obtiveram registros satisfatórios ${ }^{28}$.

O governo do Ceará, desde o final da década de 1980, bem como outros Estados do Nordeste passaram a investir na expansão do agronegócio, estimulando o aumento da utilização intensiva dos agrotóxicos, tanto por parte das grandes empresas agrícolas como pela agricultura familiar e de subsistência ${ }^{29}$. Com isso, essa população fica mais vulnerável em razão do maior acesso a pesticidas, o que pode desencadear transtornos psiquiátricos importantes, conforme discutido anteriomente.

Em relação à tendência crescente das lesões autoprovocadas no grupo etário de 10 a 19 anos, esta pode ser explicada pela intensa fase de conflito pela qual o jovem passa. Em outros estudos, resultados denotam a adolescência como a etapa com maior índice de comportamento suicida, com médias etárias variando entre 12 e 15 anos, uma vez que as manifestações do crescimento do adolescente assemelham-se à formação de sintomas neuróticos, psicóticos ou de personalidade antissocial, sendo normal nessa fase a ocorrência de comportamento inconsistente e imprevisível por algum tempo. Esse contexto contribui para o aparecimento de modos destrutivos de lidar com a realidade e, inclusive, de distúrbios psiquiátricos diversos no jovem ${ }^{30}$.

A constante busca por identidade, os amores não correspondidos, os conflitos familiares decorrentes de sua mu- dança de comportamento, a inquietude e medo diante da vida que se descortina e a intensa instabilidade emocional acabam por induzir o jovem à autoagressão, muitas vezes somente para chamar a atenção da família ${ }^{31}$. Do total de óbitos registrados no Brasil, 1\% decorre de suicídios, sendo que, em indivíduos registrados entre 15 e 29 anos de idade, essa proporção atinge $4 \%$ do total de mortes ${ }^{5}$. Segundo Teti et al..$^{32}$, adolescentes mais novos tendem a ter menos maturidade emocional para resolução dos problemas cotidianos, como brigas familiares, relações afetivas, assuntos escolares, agindo com maior impulsividade ao pensar em medidas drásticas como suicídio.

A agressão sexual é um importante fator a ser considerado acerca da tendência dos casos de violência autoprovocadas no Brasil, que, apesar da alta incidência e da indignação que causa na sociedade, ainda é uma das agressões de maior subnotificação e sub-registro. Esses eventos abalam emocionalmente o indivíduo, que passa a desenvolver distúrbios psiquiátricos importantes, como a depressão e a ansiedade, aumentando o risco para a morte. Presente em todas as classes sociais, etnias e culturas, a violência sexual ainda conta com um número muito pequeno de denúncias. Os danos e sequelas resultantes desse tipo de agressão constituem um importante agravo em todas as sociedades e países, porém vários fatores colaboram para o pouco conhecimento epidemiológico sobre eles ${ }^{31}$.

Estudos chamavam a atenção para a problemática da criança pobre, abandonada, negligenciada, drogada, prostituída, sem escola, sem lar, sem comida, vítima de maus-tratos físicos e/ou de abuso sexual, havendo necessidade de melhorar os dados de morbidade em relação à violência infantil. Os autores ainda destacam a importância da intervenção o mais precoce possível, desde a prevenção até a punição de atos abusivos, tornando realidade o Estatuto da Criança e do Adolescente por meio da identificação e notificação dos $\operatorname{casos}^{33}$. A Nature Neuroscience, por intermédio de uma publicação em março de 2009, demonstrou que o abuso sexual e físico, especialmente nos primeiros anos de vida, aumenta a quantidade de um tipo de receptor no cérebro de suicidas que se localiza numa importante via neuroendócrina do organismo, cuja sua principal função é regular a resposta comportamental e fisiológica aos eventos estressores. Com mais receptores, a resposta é amplificada ${ }^{34}$.

Desse modo, o apoio psicológico e familiar, nessa fase, é essencial para que o jovem descubra suas aptidões e oportunidades. Atravessar essa fase da vida de maneira saudável no mundo atual torna-se um desafio, mas possível diante dos inúmeros recursos que a sociedade globalizada oferece. Tratamentos específicos devem ser considerados para aqueles que experimentam a depressão nessa fase do crescimento. A escola, a comunidade e a família devem estar atentas nesse sentido ${ }^{31}$. 
Acerca do aumento do coeficiente de mortalidade por LAl no grupo etário de 20 a 59 anos, trata-se de uma tendência mundial. Mello-Santos et al. ${ }^{35}$ sinalizam que, sendo esse grupo etário a população economicamente ativa e diretamente ligada ao mercado de trabalho, acaba se submetendo às condições socioeconômicas desfavoráveis, o que resulta em maior mortalidade entre os homens jovens. Estudos desenvolvidos em países do ocidente e oriente relataram resultados similares relacionados às crises e às oscilações econômicas, desencadeadas pelo desemprego e sofrimento psíquico agravado por outras configurações sociais como o patriarcado ${ }^{36}$.

Apesar de não identificarmos neste estudo uma tendência de crescimento para LAI na população de idosos, o suicídio na terceira idade também se constitui como um fenômeno importante a ser investigado para sua melhor compreensão em estudos futuros, levando em consideração que tal população é a que mais cresce, tanto no Brasil quanto na maior parte do mundo. De acordo com Baptista et al. ${ }^{37}$, as taxas de suicídio em pessoas idosas obtiveram um aumento quando comparadas à população jovem, que realiza mais tentativas de suicídio, uma vez que idosos tendem a comunicar menos suas ideações, bem como são mais letais em seus comportamentos suicidas.

Alguns fatores limitaram a abrangência dos estudos acerca de lesões autoprovocadas e merecem ser considerados, sobretudo a ausência de dados municipais e estaduais sobre vários aspectos e mais informações sobre condutas autodestrutivas. Em geral, os dados de mortalidade nos países subdesenvolvidos tendem a ser deficientes e, com essa precariedade de informações sobre suicídio com base nacional, fica difícil o estabelecimento de comparações com outros estados e países. Nesse sentido, a World Health Organization $(\mathrm{WHO})^{4}$ afirma que a variabilidade das taxas de suicídio por idade é ainda maior que aquela que existe segundo regiões do mundo. A subnotificação dos dados é outro fator que prejudica o entendimento, uma vez que a proporção de "causas mal definidas" é elevada ${ }^{38}$.

Além de sub-registro e de subnotificação, há o problema dos suicídios que "se escondem" sob outras denominações de causa de morte, como, por exemplo, acidente automobilístico, afogamento, envenenamento acidental e "morte de causa indeterminada"39.

\section{CONCLUSÕES}

Os resultados apontam que, no período de 2004 a 2014, houve crescimento do coeficiente de mortalidade por lesões autoprovocadas intencionalmente no Brasil e em todas as cinco regiões que compõem o cenário nacional. No que se refere à faixa etária, o grupo compreendido entre 10 a 19 anos foi o que apresentou maior coeficiente de mortalidade, seguido da faixa etária de 20 a 59 anos e de maiores de 60 anos.

Evidencia-se a necessidade de maior conhecimento dos mecanismos socioculturais e econômicos que, direta e/ou indiretamente, constituem os mecanismos das lesões autoprovocadas intencionalmente nas diferentes regiões do Brasil, sendo de fundamental importância para a formulação de políticas públicas específicas que visem contribuir para a redução da tendência desse tipo de mortalidade.

Diante da complexidade das causas e consequências da violência, sugerem-se novos estudos que possam preencher as lacunas de conhecimento do fenômeno do suicídio, no sentido de melhor compreendê-lo em cada região brasileira, não o reduzindo ao plano individual, mas sim adotando medidas interdisciplinares e específicas na sua prevenção.

\section{CONTRIBUIÇÕES INDIVIDUAIS}

\section{Lélia Lessa Teixeira Pinto, Saulo Sacramento Meira} e Ícaro José Santos Ribeiro - Contribuíram significativamente na concepção e elaboração do estudo, sendo a análise, interpretação dos dados, redação do artigo, revisão crítica do conteúdo intelectual e aprovação da versão final a ser publicada.

Adriana Alves Nery e Cezar Augusto Casotti - Contribuíram significativamente na concepção do estudo, orientação, correção, revisão crítica do conteúdo intelectual e aprovação da versão final a ser publicada.

\section{CONFLITOS DE INTERESSE}

Os autores não possuem conflitos de interesse a serem declarados.

\section{REFERÊNCIAS}

1. Krug EG, Mercy JA, Dahlberg LL, Zwi AB. World report on violence and health. Geneva: World Health Organization; 2002.

2. Crosby AE, Ortega L, Melanson C. Self-directed violence Surveillance: Uniform Definitions and Recommended Data Elements, Version 1.0. Atlanta (GA): Centers for Disease Control and Prevention, National Center for Injury Prevention and Control; 2011

3. CBCD - Centro Colaborador da OMS para a Classificação de Doenças em Português (Centro Brasileiro de (lassificação de Doenças). Classificação Estatística Internacional de Doenças e Problemas Relacionados à Saúde. São Paulo, Brasilia: Faculdade de Saúde Pública da Universidade de São Paulo, Organização Mundial de Saúde, Organização Pan-Americana de Saúde; 2017. Disponível em: http://www.datasus.gov.br/cid10/v2008/cid10.htm.

4. WHO - World Health Organization. Preventing suicide: a global imperative. Luxembourg: WHO; 2014.

5. Brasil. Ministério da Saúde. Secretaria de Vigilância em Saúde. Departamento de Análise de Situação em Saúde. Saúde Brasil 2012: uma análise da situação de saúde e dos 40 anos do Programa Nacional de Imunizações/Ministério da Saúde, Secretaria de Vigilância em 
Saúde, Departamento de Análise de Situação em Saúde. Brasília: Editora do Ministério da Saúde; 2013.

6. Machado DB, Santos DN. Suicídio no Brasil, de 2000 a 2012. J Bras Psiquiatr. 2015;64(1):45-54.

7. Brzozowski FS, Soares GB, Benedet J, Boing AF, Peres MA. Suicide time trends in Brazil from 1980 to 2005. Cad. Saúde Pública. 2010;26(7):1293-302.

8. Botega NJ. Comportamento suicida: epidemiologia. Psicologia USP. 2014;25(3):231-6.

9. Värnik P. Suicide in the world. Int J Environ Res Public Health. 2012:9:760-71.

10. Durkheim E. 0 suicídio. São Paulo: Martins Fontes; 2000.

11. Viana GN, Zenkner F de M, Sakae TM, Escobar BT. Prevalência de suicídio no Sul do Brasil, 2001-2005. J Bras Psiquiatr. 2008; 57(1):38-43.

12. Stallones $L$, Beseler C. Pesticide poisoning and depressive symptoms among farm residents. Ann Epidemiol. 2002;12:389-94.

13. Rogers JR. Theoretical grounding: 'the missing link' in suicide research. J Counsel Dev. 2001;79:16-29.

14. Londres F. Agrotóxicos no Brasil: um guia para ação em defesa da vida. Rio de Janeiro: AS-PTA; 2011

15. Silva SMS. Intoxicações por inibidores da acetilcolinesterase: etiologia, diagnóstico e tratamento. Coimbra. Dissertação [Mestrado Integrado em Medicina] - Faculdade de Medicina da Universidade de Coimbra; 2015.

16. Singhal A, Ross J, Seminog 0, Hawton K, Goldacre MJ. Risk of self-harm and suicide in people with specific psychiatric and physical disorders: comparisons between disorders using English national record linkage. J R Soc Med. 2014;107(5):194-204.

17. Faria NMX, Facchini LA, Fassa AG, Tomasi E. Estudo transversal sobre saúde mental de agricultores da Serra Gaúcha (Brasil). Rev Saúde Pública. 1999;33(4):391-400

18. Marín-León L, Oliveira HB, Botega NJ. Suicide in Brazil, 2004-2010: the importance of small counties. Rev Panam Salud Publica. 2012;32(5):351-9.

19. Souza MLP, Deslandes SF, Garnelo L. Modos de vida e modos de beber de jovens indígenas em um contexto de transformações. Ciênc Saúde Coletiva. 2010;15(3):709-16.

20. Souza MLP, Orellana JDY. Suicide mortality in São Gabriel da Cachoeira, a predominantly indigenous Brazilian municipality. Rev Bras Psiquiatr. 2012;34:34-7.

21. World Health Organization. The ICD-10 Classification of Mental and Behavioural Disorders. Geneva: WHO; 2004.

22. Coimbra Junior CEA, Santos RV, Escobar AL (Orgs.). Epidemiologia e saúde dos povos indígenas no Brasil. Rio de Janeiro: Editora Fiocruz; 2003.

23. Almasi K, Belso N, Kapur N, Webb R, Cooper J, Hadley S. Risk factors for suicide in Hungary: A case-control study. BMC Psychiatry. 2009;9:1-9.
24. Moreira N, Gonçalves R. Perturbação mental e ideação suicida entre reclusos preventivos. Aná Psicológica. 2010;1(28):133-48.

25. Dejours C. A banalização da injustiça social. 5a ed. Rio de Janeiro: Ed. FGV; 2003.

26. Teixeira JRB. Ferraz CEl de 0, Couto Filho JCF, Nery AA, Casotti CA. Intoxicações por agrotóxicos de uso agrícola em estados do Nordeste brasileiro, 1999-2009. Epidemiol Serv Saúde. 2014;23(3):497-508.

27. Rockett IRH, Smith GS. Suicide misclassification in an international context. Atlanta: Centers for Disease Control and Prevention; 1995.

28. Andrade CLT, Szwarcwald CL. Desigualdades sócio-espaciais da adequação das informações de nascimentos e óbitos do Ministério da Saúde, Brasil, 2000-2002. Cad Saúde Pública. 2007;23(5):1207-16

29. Castro MGGM, Ferreira AP, Mattos IE. Uso de agrotóxicos em assentamentos de reforma agrária no município de Russas (Ceará, Brasil): um estudo de caso. Epidemiol Serv Saude. 2011;20(2):245-54

30. Carmona A, Arango A, Castãno J, Escobar J, García C, Godoi S. Caracterización del intento de suicidio en una población ingresada a um hospital infantil de Manizades (Caldas, Colômbia), 2000-2008. Archivos de Medicina. 2010;10(1):9-18.

31. Martins CBG, Andrade SM. Violência na infância e juventude: atendimentos hospitalares e mortalidade em município da Região Sul do Brasil. Acta Sci Health Sci (Maringá). 2006;28(1):29-35.

32. Teti GL, Rebok F, Rojas SM, Grendas L, Daray FM. Systematic review of risk factors for suicide and suicide attempt among psychiatric patients in Latin America and Caribbean. Rev Panam Salud Publica. 2014;36(2):124-33.

33. Gonçalves HS, Ferreira AL. A notificação da violência intrafamiliar contra crianças e adolescentes por profissionais de saúde. Cad Saúde Pub. 2002;18(1):315-9.

34. Christante L. Com (sem) saída. Unespciência. 2010; p. 30-5.

35. Mello-Santos C, Bertolote JM, Wang Y. Epidemiology of suicide in Brazil (1980-2000): characterization of age and gender rates of suicide. Rev Bras Psiquiatr. 2005;27(2):131-4.

36. Lin JJ, Lu TH. Suicide mortality trends by sex, age and method in Taiwan, 1971-2005. BMC Public Health. 2008;8(6):35.

37. Baptista MN, Morais PR, Rodrigues T, Silva JAC. Correlação entre sintomatologia depressiva e prática de atividades sociais em idosos. Aval Psicol. 2006; 5(1):77-85.

38. Pinto LW, Assis SG. Estudo descritivo das tentativas de suicídio na população idosa brasileira, 2000 - 2014. Ciênc Saúde Coletiva. 2015;20(6):1681-92.

39. Gotsens M, Marí-Dell'Olmo M, Rodríguez-Sanz M, Martos D, Espelt A, Pérez Glòria, et al. Validación de la causa básica de defunción en las muertes que requieren intervención medicolegal. Rev Esp Salud Publica. 2011;85(2):163-74. 